\title{
Clinical Outcomes of Emergent Laparotomies in Hypotensive Patients: 9-years Experience at a Single Level 1 Trauma Center
}

\author{
Jaeri Yoo, Byung Hee Kang * \\ Division of Trauma Surgery, Department of Surgery, Ajou University School of Medicine, Suwon, Korea
}

\section{Article history:}

Recevied: March 31, 2021

Revised: May 28, 2021

Accepted: May 28, 2021

\begin{abstract}
Purpose: The prognosis of an emergent laparotomy in hypotensive patients is poor. This study aimed to review the outcomes of hypotensive patients who had emergent laparotomies and elucidate the risk factors of mortality.

Methods: Patients who underwent an emergent laparotomy from January 2011 to December 2019 were retrospectively reviewed. The exclusion criteria included initial systolic blood pressure $>90$ $\mathrm{mmHg}$, aged < 19 years, and cardiac arrest before the laparotomy. Patients were categorized into survival groups (survived or deceased). Univariate and multivariate analyses were conducted to determine the risk factors of mortality. The time from the laparotomy to death was also reviewed and the effect of organ injury.

Results: There were 151 patient records, analyzed 106 survivors, and 45 deceased. The overall mortality was $29.8 \%$. Liver injury was the main organ-related event leading to an emergent laparotomy, and most patients died in the early phase following the laparotomy. Following multivariate analysis, the Glasgow Coma Scale score [odds ratio (95\% confidential interval) 0.733 (0.586-0.917), $p=0.007$ ], total red blood cell transfusion volume in 24 hours[1.111 (1.049-1.176), $p<$ 0.001 ], major bleed from the liver [3.931 (1.203-12.850), $p=0.023$ ], and blood lactate [1.173 (1.009$1.362), p=0.037$ ] were identified as risk factors for mortality.

Conclusion: Glasgow Coma Scale score, total red blood cell transfusion volume in 24 hours, major bleed from the liver, and lactate were identified as risk factors for mortality. Initial resuscitation and management of liver injuries have major importance following trauma.
\end{abstract}

Keywords: hypotension, laparotomy, risk factors, treatment outcome, wounds and injuries

\author{
${ }^{*}$ Corresponding Author: \\ Department of Surgery, Ajou University \\ School of Medicine, 164 Worldcup-ro, \\ Yeongtong-gu, Suwon, Gyeonggi, 16499, \\ E-mail: kbhname@gmail.com \\ ORCID \\ JaeriYoo \\ https://orcid.org/0000-0003-0370-6685 \\ Byung Hee Kang \\ https://orcid.org/0000-0003-3204-3251
}

$21 \%$. However, the mortality rate for hypotensive patients appeared unchanged at $46 \%$, despite improvements in trauma care [4].

In the Republic of Korea in 2012, the government implemented the use of regional trauma centers. Since trauma patients are directed to a regional trauma center, it is easier to collect patient data, and the time taken to start an emergent laparotomy is monitored in hypotensive patients.

Recent studies from 1 of these trauma centers have reported that mortality after damage control surgery was about $42 \%$ $[5,6]$, which was similar to studies in the USA $[3,4]$. Mun et al also reported that mortality after a trauma laparotomy in 
major trauma patients was 23.7\% [7]. Ajou University Medical Center, Suwon, Korea currently acts as a regional trauma center, enabling the collection of patient data using the Korean Trauma Data Bank. The purpose of this study was to review the outcome of patients with hypotensive trauma who had an emergent laparotomy and to elucidate the risk factors associated with mortality.

\section{Materials and Methods}

This study was reviewed and approved by the appropriate institutional review board (no.: AJIRB-MED-MDB-20-581). This study included trauma patients who underwent an emergent laparotomy from January 2011 to December 2019. The exclusion criteria were as follows: initial systolic blood pressure $(\mathrm{SBP})>90 \mathrm{mmHg}$, aged < 19 years, and cardiac arrest before laparotomy. The patients included in this retrospective review were categorized into 2 groups according to whether they survived or deceased. Patient data regarding their initial physiological parameters, general characteristics, injury severity, and treatment was also collected. Injury-to-operation time was defined as "the time from injury to the first surgical incision," and operation duration was defined as "the time from the first surgical incision to closure" (including temporary closure).

Comparisons between the groups were performed using the Student $\mathrm{t}$ test or the Mann-Whitney U test (after normality tests for continuous variables). Variables were presented as mean \pm standard deviation for the Student $t$ test, and as the median (interquartile) for the Mann-Whitney U test. The Chisquare test or the Fisher's exact test was used for categorical variables. Following univariate analysis, variables with $p<$ 0.05 were included in the multivariate analysis by applying binary logistic regression. The results of the binary logistic analyses were summarized by estimating the odds ratios and the respective 95\% confidence intervals. All analyses were performed using IBM SPSS Version 21.0 (IBM Corp., Armonk, NY, USA).

\section{Results}

\section{Patient characteristics}

Amongst the 908 patients who underwent an emergent laparotomy, there were 151 patients who were included in the analysis. Of these, 106 patients survived and 45 died. The overall mortality rate was $29.8 \%$ (Figure 1 ). Overall, the Trauma and Injury Severity Score was 61.5\%. Amongst the 45 deceased patients, 19 (42.2\%) died due to a hemorrhage (all within 24 hours of surgery), 20 (44.4\%) died from organ failure, and 6 (13.3\%) died from severe brain damage. Compared with the group that survived, the deceased group displayed a lower SBP $(75.1 \pm 13.0$ vs $63.5 \pm 20.0, p<0.001)$, a lower Glasgow Coma Scale (GCS) score ( $11.7 \pm 3.9$ vs $6.0 \pm 4.5, p<0.001)$, and a higher lactate level ( $5.2 \pm 3.3$ vs $8.5 \pm 3.8, p<0.001$; Table 1$)$.

Table 1. General patients' characteristics on admission.

\begin{tabular}{|c|c|c|c|}
\hline & $\begin{array}{l}\text { Survived } \\
(n=106)\end{array}$ & $\begin{array}{l}\text { Deceased } \\
(n=45)\end{array}$ & $p$ \\
\hline Age (y) & $48.7 \pm 15.5$ & $48.4 \pm 15.2$ & 0.921 \\
\hline Sex (male: female) & $82: 24: 00$ & $35: 10: 00$ & 0.955 \\
\hline \multicolumn{4}{|l|}{ Mechanism } \\
\hline Blunt & $93(87.7 \%)$ & $43(95.6 \%)$ & 0.233 \\
\hline Penetrating & $13(12.3 \%)$ & $2(4.4 \%)$ & \\
\hline \multicolumn{4}{|l|}{ Admission vital sign } \\
\hline Systolic blood pressure $(\mathrm{mmHg})$ & $75.1 \pm 13.0$ & $63.5 \pm 20.0$ & 0.001 \\
\hline Pulse (beats/min) & $97.2 \pm 27.4$ & $104.3 \pm 37.1$ & 0.198 \\
\hline Glasgow coma scale score & $11.7 \pm 3.9$ & $6.0 \pm 4.5$ & $<0.001$ \\
\hline Revised trauma score & $5.9 \pm 1.6$ & $3.9 \pm 1.9$ & $<0.001$ \\
\hline Transfer from other hospitals & $64(60.4 \%)$ & $23(51.1 \%)$ & 0.292 \\
\hline Initial lactate (mmol/L) & $5.2 \pm 3.3$ & $8.5 \pm 3.8$ & $<0.001$ \\
\hline
\end{tabular}




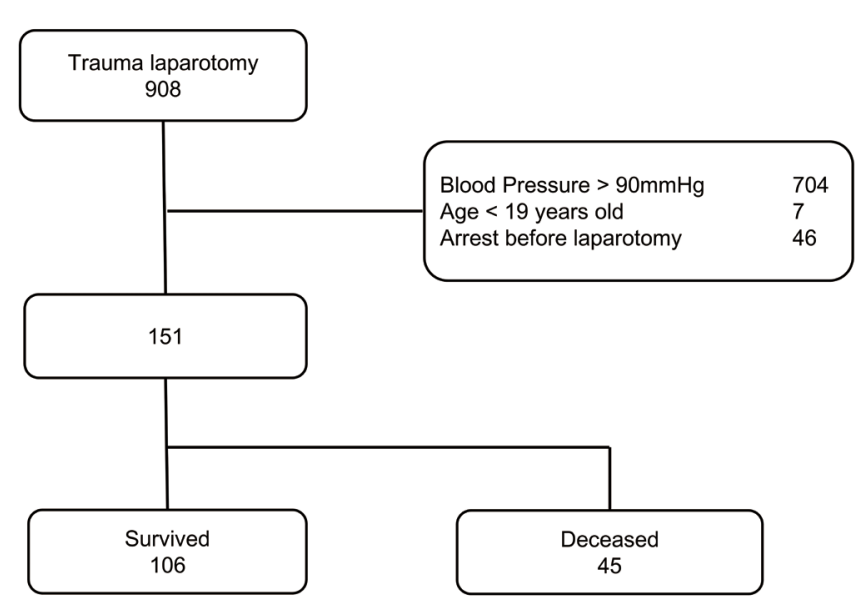

Figure 1. Flowchart of study inclusion.

\section{Injury severity and outcome of laparotomy}

Using the Abbreviated Injury Scale (AIS), the liver was the most common major organ injury, and was also considered the most life-threatening factor. Amongst hypotensive patients with a major bleed from the liver, $55.3 \%$ of patients died. No mortality was observed in those with injuries to the pancreas, colon, abdominal wall, or bladder (Figure 2).

The deceased group experienced more severe injuries and required higher volumes of transfused red blood cells (RBC), however, there was no significant difference in the time from injury to operation or admission to operation (Table 2 ) between the groups. Most patients in the deceased group died within 24 hours (Figure 3A). There were 19 patients who died due to a hemorrhage, 2 patients died from organ failure, and 1 patient died from severe brain damage. Of those who died within 24 hours of surgery, 9 (40.9\%) patients died within 1 hour of the operation (Figure 3B).

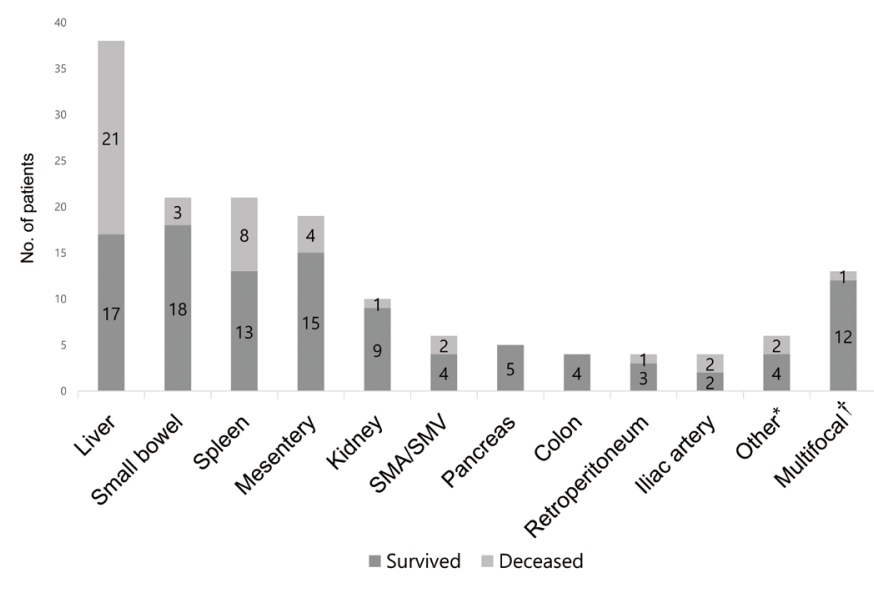

Figure 2. Mortality according to the highest Organ Injury Scale scores. * Other: Duodenum, bladder, rectum, and abdominal wall. $\dagger$ Multifocal: Injuries of the highest grade for more than 2 organs. SMA = superior mesenteric artery; SMV = superior mesenteric vein.

Table 2. Injury characteristics of laparotomy.

\begin{tabular}{|c|c|c|c|}
\hline & Survived $(n=106)$ & Deceased $(n=45)$ & $p$ \\
\hline Injury severity score & $25(18-34)$ & $34(25-50)$ & 0.001 \\
\hline \multicolumn{4}{|l|}{ Abbreviated injury scale score } \\
\hline Head & $3(2-4)$ & $4(3-5)$ & 0.121 \\
\hline Thorax & $3(3-3)$ & $3(3-4)$ & 0.385 \\
\hline Abdomen & $4(3-4)$ & $4(4-5)$ & 0.026 \\
\hline Extremity & $3(2-3)$ & $3(2-5)$ & 0.174 \\
\hline Major bleed from the liver * & $19(17.9 \%)$ & $21(46.7 \%)$ & $<0.001$ \\
\hline TRISS & $86.7(58.8-95.3)$ & $20.8(6.5-60.6)$ & $<0.001$ \\
\hline \multicolumn{4}{|l|}{ Transfusion in $24 \mathrm{~h}$} \\
\hline Packed red blood cell (U) & $11(5-19)$ & $34(24-53)$ & $<0.001$ \\
\hline Fresh frozen plasma $(U)$ & $10(5-15)$ & $22(10-38)$ & $<0.001$ \\
\hline Injury-to-operation time (min) & $212(159-295)$ & $210(136-280)$ & 0.591 \\
\hline Admission-to-operation time (min) & $101(66-144)$ & $98(61-125)$ & 0.241 \\
\hline Operation duration (min) & $100(80-139)$ & $100(65-125)$ & 0.2 \\
\hline Temporary abdomen closure & $65(61.3 \%)$ & $37(82.2 \%)$ & 0.012 \\
\hline
\end{tabular}

* Liver was highest AIS among injured organ.

TRISS = trauma and injury severity score. 
(A) 25

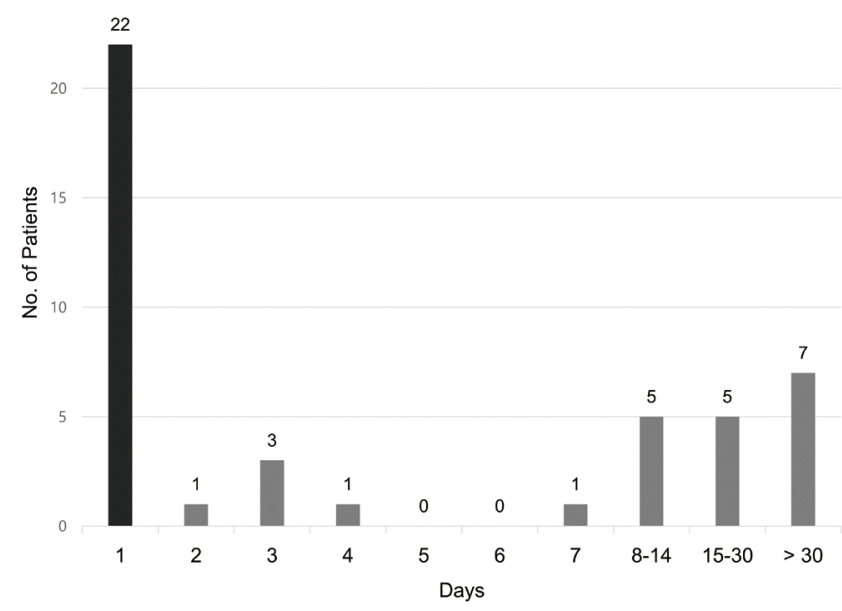

(B) 10

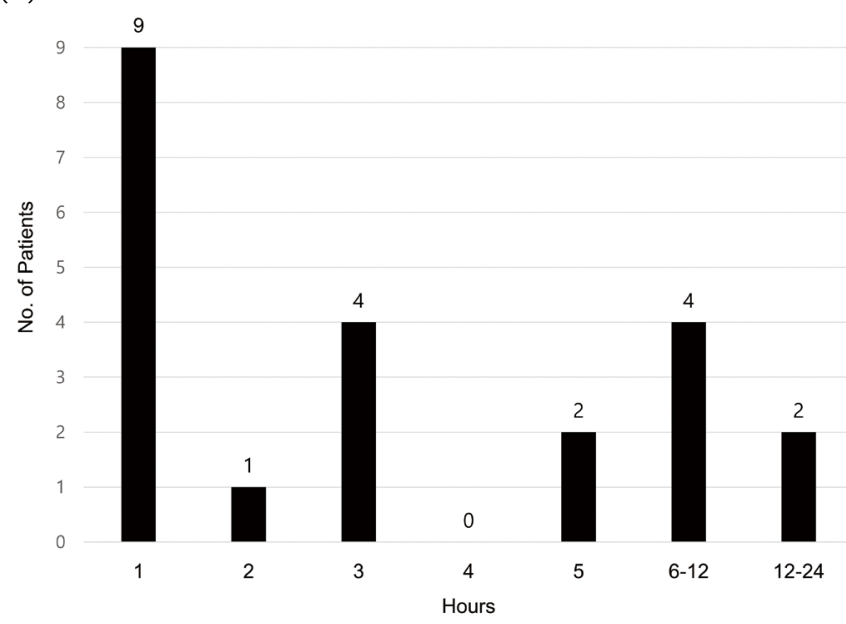

Figure 3. Timing of death (A) days after admission following a laparotomy; (B) hours within the first day.

\section{Risk factors of mortality after laparotomy in hypotensive patients}

Univariate analysis was applied to the GCS score, Revised Trauma Score, Injury Severity Score, a major bleed from the liver, total RBC and fresh frozen plasma (FFP) transfusion volumes within 24 hours, lactate levels, and the percentage undergoing temporary abdomen closure were all selected for multivariable analysis. Following multivariate analysis, the GCS score [odds ratio (95\% confidence interval): 0.733 (0.586$0.917), p=0.007$ ], total RBC transfusion volume in 24 hours [1.111 (1.049-1.176), $p<0.001$ ], major bleeding from liver [3.931 (1.203-12.850), $p=0.023$ ], and elevated blood lactate [1.173 (1.009-1.362), $p=0.037$ ] demonstrated significant differences between the survived and deceased groups (Table 3 ).

\section{Discussion}

Emergent laparotomies in hypotensive patients following trauma is associated with a high mortality rate. Understanding the risk factors associated with mortality, which are yet to be fully elucidated, is of the utmost importance. In this retrospective study, the overall mortality of $29.8 \%$ was lower than the rates observed in previous studies in the USA [3,4], and Korea [5] (40-46\%)

Among the deceased group, more than half of the patients died within a day after the laparotomy, and most of these deaths occurred within 5 hours. Hemorrhages are the leading cause of death following trauma, so emphasis is on early hemorrhagic control $[5,8]$. However, the debate as to whether

Table 3. Univariate and multivariate analyses for risk factors of mortality.

\begin{tabular}{|c|c|c|c|c|}
\hline & Univariate analysis & $p$ & Multivariate analysis & $p$ \\
\hline GCS score & $0.756(0.687-0.830)$ & $<0.001$ & $0.733(0.586-0.917)$ & 0.007 \\
\hline $\mathrm{RBC}$ in $24 \mathrm{~h}$ & $1.091(1.058-1.125)$ & $<0.001$ & $1.111(1.049-1.176)$ & $<0.001$ \\
\hline Major bleed from the liver* & $4.007(1.859-8.633)$ & $<0.001$ & $3.931(1.203-12.850)$ & 0.023 \\
\hline Lactate & $1.293(1.149-1.453)$ & $<0.001$ & $1.173(1.009-1.362)$ & 0.037 \\
\hline ISS & $1.049(1.021-1.077)$ & 0.001 & $1.018(0.976-1.062)$ & 0.406 \\
\hline TAC & $3.995(1.552-10.284)$ & 0.004 & $1.181(0.285-4.888)$ & 0.819 \\
\hline FFP in $24 \mathrm{~h}$ & $1.055(1.027-1.084)$ & $<0.001$ & $0.956(0.904-1.010)$ & 0.107 \\
\hline RTS & $0.543(0.434-0.697)$ & $<0.001$ & $1.365(0.711-2.418)$ & 0.286 \\
\hline
\end{tabular}

Hosmer-Lemeshow goodness-of-fit ( $\mathrm{DF}=8$ ) Chi-square 13.704, $p=0.090$.

${ }^{*}$ Liver was highest AIS among injured organ.

AIS = abbreviated injury scale; FFP = fresh frozen plasma; GCS = glasgow coma scale; ISS = injury severity score; RBC = red blood cell; RTS = revised trauma score; SBP = systolic blood pressure; TAC = temporary abdomen closure. 
time to laparotomy is an important factor for survival is ongoing. Barbosa et al demonstrated that an increased time to operation was associated with mortality in face, arm, speech test positive patients [9]. In contrast, Undurraga Perl et al [10] reported that the resuscitation strategy, including time to laparotomy, did not affect survival. Similarly, Groven et al [11] reported that an increased time to laparotomy did not increase mortality. In the current study, a difference in time to laparotomy between the survival and deceased groups was not observed. However, unlike other studies where the time to a laparotomy was around 30-40 minutes, in this current study approximately 100 minutes elapsed between admission and the operation. The implementation of regional trauma centers began in 2012 in Republic of Korea, and the trauma center in Ajou University Medical Center, Suwon, Korea was built 2016. It seemed that the trauma system of care had been slow to be adopted in Korea compared with other countries. Clarke et al [3] demonstrated that the time to a laparotomy did affect survival, but only for delays up to 90 minutes. Therefore, the time to a laparotomy in the current study was possibly too long (100 minutes) to impact survival, and an increased effort to shorten this time is required.

This current study did reveal a significant difference in the GCS score between the surviving and deceased groups. The GCS score is a well-known predictor of survival in patients with head trauma and overall trauma [12,13]. Moreover, it can be used as a predictor of mortality in patients with pelvic trauma or for those patients who undergo damage-control laparotomy $[14,15]$. Consistent with these studies, following multivariate analysis, the results of this study showed that a statistically lower GCS score was a risk factor of mortality between the 2 groups, despite there being no statistical difference observed in the AIS score for the head. The GCS score reflects cerebral perfusion, which could potentially be reduced by hemorrhagic shock. Therefore, the GCS may be a stronger predictor of the probability of survival than SBP, respiratory rate, and even the Injury Severity Score [16,17].

The amount of blood transfusion required for a patient is related to the extent of bleeding, therefore, the total RBC transfusion volume in 24 hours was also a risk factor in the current study. However, the amount of FFP transfused in 24 hours was not a risk factor following multivariate analysis. In addition to the total transfusion volume (the FFP to RBC transfusion ratio) was also important in trauma patients who received massive blood transfusion. Roquet et al demonstrated that an FFP:RBC ratio of more than 1:1.5, transfused within 6 hours, improved the 30-day mortality rate in trauma patients with severe bleeding [18]. Although the FFP to RBC ratio was not specifically reviewed in this study, the ratio was higher in the survival group, at almost $1: 1$ compared with $1: 1.5$ in the deceased group.
Regarding the effects of organ injury, even when using the same injury scale, the rate of mortality is different depending on the damaged organ. Damage to the liver shows the poorest outcome among Grade IV-V injuries [19]. Similarly, in this current study, more than half of the patients with a major bleed in the liver had deceased. Management of severe liver injuries is challenging but has improved with the adoption of a multidisciplinary approach [20,21]. Grade IV-V injuries to the pancreas or colon have been associated with a mortality rate of $28-32 \%$ [19], however, in the current study in patients with injuries to the pancreas and colon no mortality occurred. This may be because the number of patients with pancreas and colon injuries was small and the highest injury grade was Grade III.

This study had several limitations. Firstly, the study design was retrospective, and the number of patients was too small for meaningful epidemiological analysis. Secondly, during the 9-year study period, trauma care has improved through the implementation of trauma centers within hospitals. Thirdly, main abdomen organ injury was presented in this study but multiple lower grade abdomen organ injuries were excluded from analysis. In conclusion, emergent laparotomies in hypotensive patients resulted in a high mortality rate. The GCS score, total RBC transfusion volume in 24 hours, a major bleed from the liver, and blood lactate were determined to be significant risk factors for mortality. Furthermore, mortality mostly occurred within 24 hours of the laparotomy, hence, early control of bleeding is important and could reduce the total RBC transfusion, which is a risk factor of mortality. Management of liver injuries remains a challenge in trauma laparotomy.

\section{Conflicts of Interest}

The authors declare no conflicts of interest to declare.

\section{References}

[1] Cannon JW. Hemorrhagic Shock. N Engl J Med 2018;378(4):370-9.

[2] Weber DG, Bendinelli C, Balogh ZJ. Damage control surgery for abdominal emergencies. Br J Surg 2014;101(1):e109-18.

[3] Clarke JR, Trooskin SZ, Doshi PJ, Greenwald L, Mode CJ. Time to laparotomy for intra-abdominal bleeding from trauma does affect survival for delays up to 90 minutes. J Trauma 2002;52(3):420-5.

[4] Harvin JA, Maxim T, Inaba K, Martinez-Aguilar MA, King DR, Choudhry AJ, et al. Mortality after emergent trauma laparotomy: A multicenter, retrospective study. J Trauma Acute Care Surg 2017;83(3):464-8

[5] Kang WS, Jo YG, Park YC. Quality Improvement of damage control laparotomy: Impact of the establishment of a single Korean regional trauma center. World J Surg 2019;43(11):2814-21.

[6] Kang WS, Park YC, Jo YG, Kim JC. Pancreatic fistula and mortality after surgical management of pancreatic trauma: Analysis of 81 consecutive patients during 11 years at a Korean trauma center. Ann Surg Treat Res 2018;95(1):29-36 
[7] Yoo YS, Mun SP. The advantages of early trauma team activation in the management of major trauma patients who underwent exploratory laparotomy. Ann Surg Treat Res 2014;87(6):319-24.

[8] Henderson KI, Coats TJ, Hassan TB, Brohi K. Audit of time to emergency trauma laparotomy. Br J Surg 2000;87(4):472-6.

[9] Barbosa RR, Rowell SE, Fox EE, Holcomb JB, Bulger EM, Phelan HA, et al. Increasing time to operation is associated with decreased survival in patients with a positive FAST examination requiring emergent laparotomy. J Trauma Acute Care Surg 2013;75(1 Suppl 1):S48-52.

[10] Undurraga Perl VJ, Leroux B, Cook MR, Watson J, Fair K, Martin DT, et al. Damage-control resuscitation and emergency laparotomy: Findings from the PROPPR study. J Trauma Acute Care Surg 2016;80(4):568-74; discussion 574-5.

[11] Groven S, Naess PA, Skaga NO, Gaarder C. Effects of moving emergency trauma laparotomies from the ED to a dedicated OR. Scand J Trauma Resusc Emerg Med 2013;21:72.

[12] Cevik AA, Abu-Zidan FM. Searching for mortality predictors in trauma patients: A challenging task. Eur J Trauma Emerg Surg 2018;44(4):561-5.

[13] Champion HR, Sacco WJ, Copes WS, Gann DS, Gennarelli TA, Flanagan ME. A revision of the Trauma Score. J Trauma 1989;29(5):623-9.

[14] Liao LM, Fu CY, Wang SY, Liao CH, Kang SC, Ouyang CH, et al. Risk factors for late death of patients with abdominal trauma after damage control laparotomy for hemostasis. World J Emerg Surg 2014;9:1.
[15] Wang H, Phillips JL, Robinson RD, Duane TM, Buca S, Campbell-Furtick $\mathrm{MB}$, et al. Predictors of mortality among initially stable adult pelvic trauma patients in the US: Data analysis from the National Trauma Data Bank. Injury 2015;46(11):2113-7.

[16] Kimura A, Chadbunchachai W, Nakahara S. Modification of the Trauma and Injury Severity Score (TRISS) method provides better survival prediction in Asian blunt trauma victims. World J Surg 2012;36(4):813-8.

[17] Kimura A, Nakahara S, Chadbunchachai W. The development of simple survival prediction models for blunt trauma victims treated at Asian emergency centers. Scand J Trauma Resusc Emerg Med 2012;20:9.

[18] Roquet F, Neuschwander A, Hamada S, Fave G, Follin A, Marrache D, et al. Association of early, high plasma-to-red blood cell transfusion ratio with mortality in adults with severe bleeding after trauma. JAMA Netw Open 2019;2(9):e1912076.

[19] Esposito TJ, Tinkoff G, Reed J, Shafi S, Harbrecht B, Thomas C, et al. American Association for the Surgery of Trauma Organ Injury Scale (OIS): Past, present, and future. J Trauma Acute Care Surg 2013;74(4):1163-74.

[20] Coccolini F, Coimbra R, Ordonez C, Kluger Y, Vega F, Moore EE, et al. Liver trauma: WSES 2020 guidelines. World J Emerg Surg 2020;15(1):24.

[21] Suen K, Skandarajah AR, Knowles B, Judson R, Thomson BN. Changes in the management of liver trauma leading to reduced mortality: 15-year experience in a major trauma centre. ANZ J Surg 2016;86(11):894-9. 